\title{
Retos éticos de los agentes comunicativos en situaciones de conflicto
}

\section{Ethical challenges of communicative agents in conflict situations}

\author{
Elena Alcalde Peñalver*
}

Resumen: En este artículo se presentarán los principales retos éticos a los que se enfrentan los traductores, entendiéndolos como agentes comunicativos entre dos lenguas, al realizar su labor en situaciones de conflicto. Para ello, en primer lugar, enmarcaremos la actividad que estos realizan en su contexto, definiendo las nociones principales que esta engloba y los principios éticos que deben asumir para que en su actuación a la hora de transmitir un mensaje no influyan los intereses de las partes. Seguidamente, expondremos la metodología que consideramos más oportuna para poder adoptar esta posición neutral en una situación de conflicto. Posteriormente, nos detendremos en un ejemplo determinado el que examinaremos la aplicación de todos estos aspectos en la actividad de un agente comunicativo en Irak tras los atentados del 11S, para finalizar a continuación con una serie de conclusiones.

Palabras clave: Agente comunicativo; situación de conflicto; ética de la profesión.

Abstract: In this paper, the main ethical challenges that translators acting as communicative agents between two languages face when they work in a situation of conflict will be explained. First of all we will define the main concepts of the activity they perform and the main ethical principles that should be respected so as to be able to neutrally transmit a message without being influenced by the interests of the parties. The methodology that should be followed in this case will also be explained. After that we will include a practical example of the role of a communicative agent

\footnotetext{
Departamento de Traducción e Interpretación, Universidad de Granada. Email: ealcalde@ugr.es
} 
Peñalver, E. A. - Retos éticos de los agentes comunicativos en situaciones de conflicto

in Iraq to show how all the concepts outlined in this paper can be applied. Finally, the main conclusions reached in this research paper will be explained.

Keywords: Communicative agent; situation of conflict; ethics of the profession.

\section{Introducción}

«I know you believe you understand what you think I said, but I am not sure you realize that what you heard is not what I meant». (Alan Greenspan, presidente de la Reserva Federal de Estados Unidos entre 1987 y 2006)

En el mundo globalizado en el que vivimos hoy en día, la traducción constituye un elemento clave para que las partes que estén implicadas en un conflicto puedan legitimar su versión de los hechos. Por lo tanto, una actuación ética del agente comunicativo desempeña un papel muy importante en la forma en la estos se puedan interpretar y desarrollar con posterioridad.

Sin embargo, los investigadores de la traducción han mostrado a lo largo de la corta historia de la traductología más interés en estudiar la preparación del traductor, la práctica y la traducción como producto que en investigar acerca de los efectos de la traducción como herramienta de comunicación entre dos culturas (MAIER 2007). No obstante, los acontecimientos de impacto mundial de los últimos años han hecho que se manifieste un creciente interés por investigar acerca de la función de los traductores en los medios de comunicación en tanto que agentes comunicativos que ayudan a transmitir información entre países con lenguas y culturas distintas. Así, se reconoce que la labor del traductor es más difícil cuanto más lejanas son las culturas, puesto que no se trata sólo de transmitir el significado, sino de descifrar también la connotación de los discursos (VIDAL 2010). De este modo, se ha puesto énfasis en la ética de este colectivo debido 
Peñalver, E. A. - Retos éticos de los agentes comunicativos en situaciones de conflicto

al efecto que el mensaje que transmita puede tener en los resultados de los hechos.

Este interés se ha expresado entre una mezcla de controversia y desconfianza hacia el trabajo de estos agentes comunicativos a la hora de transmitir toda la información del mensaje de forma fiel. En efecto, la labor del traductor en tanto que agente comunicativo parece estar siempre bajo sospecha y se considera que la fidelidad de la traducción con respecto a su original es una característica ambivalente del mensaje que transmiten.

En este artículo pretendemos analizar los principales retos éticos a los que se tienen que enfrentar los traductores, entendiéndolos como agentes comunicativos entre dos lenguas, al realizar su labor en situaciones de conflicto. Para ello, en primer lugar, enmarcaremos la actividad que estos realizan en su contexto, definiendo las nociones principales que esta engloba y los principios éticos que deben asumir para que en su actuación a la hora de transmitir un mensaje no influyan los intereses de las partes. Seguidamente, expondremos la metodología que consideramos más oportuna para poder adoptar esta posición neutral en una situación de conflicto. Posteriormente, nos detendremos en un ejemplo determinado el que examinaremos la aplicación de todos estos aspectos en la actividad de un agente comunicativo en Irak tras los atentados del 11S, para finalizar a continuación con una serie de conclusiones.

\section{Contextualización del objeto de estudio}

Ante la pregunta de para qué necesitan los periodistas a los traductores e intérpretes hoy en día cuando se encuentran en situaciones de conflicto, cabe afirmar que estos últimos cumplen una función muy importante en el proceso de comunicación, sobre todo debido a que la mayoría de los conflictos existentes no se restringen a comunidades específicas que hablan 
Peñalver, E. A. - Retos éticos de los agentes comunicativos en situaciones de conflicto

una sola lengua, sino que se negocian en la esfera internacional (BAKER 2006). Nos referiremos en este artículo a los traductores e intérpretes como agentes comunicativos, puesto que coincidimos con Hatim y Mason (1997: 59) en que estas dos actividades se realizan con el mismo objetivo comunicativo:

It should not be assumed that because translating in the written and in the oral mode are known by different terms, translating and interpreting, they have little in common. Although the two activities are usually rigorously separated on translator/interpreting training programmes, there is a strong case for creating a common core of fundamental issues to do with communication strategies. Many of the ways in which language users exploit the potentialities of the language system for particular purposes are common to both the written and the spoken modes».

Empecemos definiendo el objeto de estudio de este artículo para delimitar el significado de los conceptos principales a los que nos referimos. Por un lado, se reconoce que la traducción «es un proceso interpretativo y comunicativo de reformulación de un texto que se desarrolla dentro de un contexto social» (HURTADO 1999). Con esto nos referimos a que incluso cuando se pueden superar las barreras lingüísticas, aún quedan numerosos obstáculos que hay que solventar en la comunicación transcultural. De esta forma, coincidimos con Copeland y Griggs (1985: 84) en que «aprender un idioma no sustituirá nunca al aprendizaje de la cultura y del comportamiento adecuado». Con esta afirmación volvemos de nuevo al contexto social al que apelaba Hurtado y en el que se enmarca la traducción como acto comunicativo, puesto que no hay dos idiomas que sean lo suficientemente parecidos como para considerar que representan una misma realidad social (MANDElbaum 1962). En esta línea, Benjamin Whorf (1952) afirmaba que el sistema lingüístico de todo idioma no es solo un instrumento reproductor para expresar ideas, sino que más bien es lo que da forma a las ideas. Por ello, para un traductor, aprender a traducir significa producir significados que sean aceptables para la comunidad cultural a la que pertenece el lector (ARROJo 
Peñalver, E. A. - Retos éticos de los agentes comunicativos en situaciones de conflicto

1992). De esta forma, para el comunicador transcultural, el principal objetivo de la traducción no debe consistir en encontrar una equivalencia exacta sino, tal y como afirma Douglas (1975: 42) «en evitar una confrontación entre sistemas extraños de pensamiento».

La imprecisión del mensaje puede provocar malentendidos y por consiguiente, aparentes conflictos que nos llevan a continuación a delimitar el significado de este concepto, pues partimos en la presente investigación de actos de comunicación que se van a llevar a cabo en situaciones de conflicto. De modo general, podemos caracterizar el concepto de conflicto como desacuerdos entre personas (BORISOFF y VICTOR 1991). Para aportar progresivamente mayor información a esta definición, adoptamos la propuesta por Coser (1956: 64), que incorpora en la misma el concepto de poder. De este modo, define conflicto como una «lucha sobre valores y aspiraciones a gozar de una posición, poder y recursos en la que los objetivos de los oponentes consisten en neutralizar, herir o eliminar a sus rivales». Como se puede observar, nos hemos adentrado en la definición de conflicto incorporando el concepto de poder, que viene ligado a la situación comunicativa que nos atañe, puesto que entre los distintos participantes encontraremos diferentes estatus y situaciones de poder. Esto hace referencia no solo a las posiciones sociales relativas de los interlocutores, sino también al estatus de sus respectivas lenguas: la de la mayoría dominante frente a la del grupo minoritario, ambos guiados por el mismo fin: conseguir sus objetivos mediante la negociación a través de la comunicación.

Sin embargo, la noción de poder introduce también la situación del traductor o intérprete, puesto que este también tiene su posición dentro de la jerarquía de poder que apreciamos en la interacción comunicativa cara a cara (ABRIL 2006). Anderson (1976/2002: 212) señala las principales fuentes de poder del intérprete. En primer lugar, el intérprete es un participante indispensable e insustituible, puesto que sin su intervención el intercambio comunicativo no podría desarrollarse. Por otro lado, en general, es el único 
PeÑalver, E. A. - Retos éticos de los agentes comunicativos en situaciones de conflicto

participante bilingüe y bicultural en el acto comunicativo, lo que significa que no solo controla unos recursos limitados (las lenguas) sino que de hecho tiene el monopolio sobre ellos. Esto significa, que no existe control alguno sobre el trabajo del intérprete, de manera que podría incluso traducir selectivamente y decidir el resultado del acto comunicativo y, a menos que cruzara los límites de manera muy conspicua, no habría nadie que lo advirtiera (SMIRNOv 1997: 217). Tanto es así que de hecho la percepción del intérprete y su poder cambia radicalmente cuando alguno de los interlocutores primarios (o cualquier otra persona presente, como el público o los testigos en un tribunal) conoce los dos idiomas de trabajo (ANDERSON 1976/2002). Ese monopolio y ausencia de control permiten al agente comunicativo definir su propio papel, incluso dentro de unos límites establecidos, lo que de manera instintiva conduce a los participantes primarios a asumir que el resultado de la negociación dependerá del grado de filiación del intérprete con una u otra parte (ABRIL 1996). Esta percepción puede inducir a los interlocutores a buscar la complicidad de «the man in the middle» (1976/2002: 211), por ejemplo, dirigiéndose directamente a él o introduciendo comentarios con la clara intención de que el agente comunicativo los reciba pero no los transmita.

Ante todo esto, la cuestión clave que nos planteamos es a qué funciones se limita el papel del agente comunicativo en situaciones de conflicto y hasta dónde puede llegar la reformulación o manipulación del mensaje que este trasmite. En esta cuestión desempeña la ética de la profesión un papel muy importante. De este modo, partiendo de las definiciones anteriores de nuestro objeto de estudio, introducimos el concepto de ética en la traducción, coincidiendo con Chesterman (1995: 150) en que son tres los aspectos típicos que con ella se desarrollan y que debatiremos posteriormente:

- El concepto general de lealtad a las partes implicadas en el proceso de traducción.

- El grado de libertad que es aceptable en el proceso de traducción. 
Peñalver, E. A. - Retos éticos de los agentes comunicativos en situaciones de conflicto

- El debate acerca de la invisibilidad del traductor, entendiéndolo como agente comunicativo neutral y anónimo.

En cuanto a las funciones del agente comunicativo, y basándonos en el último punto de los aspectos éticos mencionados, es necesario mencionar que no debemos confundir la labor de transmisión intercultural de un mensaje de una lengua a otra con la de la mediación o arbitraje. La mediación es fundamentalmente el arte de la convicción. En nuestro caso, se trataría de convencer a las personas o grupos que se ven envueltos en un conflicto para que pongan fin a sus diferencias. Sin embargo el agente comunicativo debe garantizar a ambas partes una posición de neutralidad, transmitiendo con exactitud la información entre los oponentes para lograr así su credibilidad. En ningún momento puede formular su opinión, por lo que debe mantener la mayor discreción sobre lo que se dice y la forma en que se comunica. Tampoco se debe confundir esta función con el arbitraje, es decir, con la intervención de una persona que oiga las posiciones de las partes que no son capaces de resolver sus diferencias y decida cuál es la mejor (BORISOFF y VICTOR 1991).

Ante este dilema, nos planteamos la cuestión de cuál es realmente el papel del agente comunicativo. Hay autores que ofrecen sugerencias para determinar los límites de los intérpretes que podemos adaptar a nuestro objeto de estudio. De esta forma, Zimman (1994: 219) recoge la opinión de una intérprete británica que afirma que «[...] the limit has been reached when it comes to making decisions for other people. They need the information but they don't need you to make up their minds for them». Cambridge (2002: 123) va más allá, e incluso enumera de forma sistemática las tareas del intérprete en los servicios públicos que configuran el modelo de la imparcialidad («the impartial model»):

Interpreters using the impartial model relay messages accurately, completely and in as closely as possible the same style as the original. They do not give personal advice or opinions; do not add or 
Peñalver, E. A. - Retos éticos de los agentes comunicativos en situaciones de conflicto

omit parts of the message; do make every effort to foster the full, accurate transfer of information; do maintain strict confidentiality. They will intervene only when they need clarification of part of a message.

En este sentido y considerando que la misión del agente comunicativo está en conseguir que la comunicación sea eficaz entre las dos partes para las que está realizando su labor, adoptamos a continuación tres de las cuatro funciones que enumera Abril (2006: 96) en función de lo que se requiera en una situación comunicativa de conflicto determinada. Lo esencial de las propuestas de la autora no reside tanto en la identificación de papeles como en el marco en el que se define la toma de decisiones que lleva al agente comunicativo a elegir un papel y, más aún, a decidir cómo y cuándo pasar de uno a otro:

- Transcodificador («conduit»): consiste en transmitir en un idioma exactamente lo emitido en otro, sin edición ni adaptación alguna del mensaje. Es el papel por defecto del intérprete, el más básico y el más habitual, es decir el que asume el intérprete a menos que detecte un potencial de conflicto relacionado con alguna barrera que no sea netamente lingüística.

- Clarificador («clarifier»): es un papel que el intérprete adopta únicamente cuando el anterior no es suficiente y los interlocutores requieren alguna intervención que facilite la comprensión. Consiste en explicar conceptos para los que no existan términos equivalentes en otro idioma o cuyos equivalentes no sean entendidos por el receptor, ajustar el registro, o bien comprobar si alguna o las dos partes están asimilando los mensajes.

- Experto o agente cultural: se refiere a una intervención con la que el intérprete proporciona el marco cultural específico en el que ha de entenderse un determinado mensaje. Solo se adopta cuando la diferencia cultural puede dificultar la comprensión o conducir a un malentendido. 
Peñalver, E. A. - Retos éticos de los agentes comunicativos en situaciones de conflicto

\section{Metodología}

Teniendo en cuenta lo tratado en el apartado anterior, pretendemos a continuación dar respuesta a la pregunta planteada acerca de cómo puede saber el agente comunicativo el papel más adecuado que debe asumir según la situación comunicativa de conflicto en la que se encuentre.

Para ello, en primer lugar abordamos la cuestión general relacionada con la ideología del contenido. En cada encargo que se les asigna, los traductores se enfrentan a una opción ética: reproducir una ideología que aparezca en el discurso o alejarse de ésta. Esta será una decisión importante como cualquier otro grupo de profesionales en la sociedad. Los traductores son responsables de los textos y mensajes que transmiten y por lo tanto, son responsables de la realidad social que crean cada vez que transmiten un mensaje de una cultura a otra (BAKER 2006). De este modo, coincidimos con Chesterman (1995: 152) en que las normas aplicables al proceso de traducción para llevar a cabo una actividad ética son las siguientes:

- Norma relacionada con las expectativas del receptor: el traductor debe llevar a cabo su trabajo de forma que se ajuste a las expectativas de los que reciben la traducción.

- Norma relacionada con los vínculos existentes entre el mensaje de la lengua origen (en lo sucesivo LO) y el mensaje de la lengua término (en lo sucesivo LT): el traductor debe llevar a cabo su trabajo de manera que se establezca un vínculo apropiado entre ambos mensajes.

- Norma comunicativa: el traductor debe llevar a cabo su trabajo de manera que se establezca una relación apropiada en la transmisión del mensaje de la LO a la LT.

En este sentido, aunque los traductores no intervengan en la resolución del conflicto puesto que su labor debe limitarse a la transmisión del mensaje entre dos lenguas, tampoco estos pueden influir negativamente debido a su 
Peñalver, E. A. - Retos éticos de los agentes comunicativos en situaciones de conflicto

actuación. Es por ello que el análisis de la situación comunicativa a la que se enfrentan se revela necesario para que estos puedan realizar su labor de la mejor manera posible. De esta forma, proponemos una adaptación del modelo comunicativo de las situaciones conflictivas de Borisoff y Victor (1991), teniendo en cuenta su aplicabilidad en la actividad comunicativa del traductor o intérprete. Nos centraremos en el punto de la evaluación como fase central en el proceso de comunicación del traductor, puesto que constituye una aproximación inicial de gran importancia para detectar dónde residen las diferencias entre los interlocutores, comprender la naturaleza de su relación y el curso del conflicto. Así, podremos definir las estrategias de comunicación adecuadas para transmitir este mensaje de forma que no afecte a su contenido, teniendo en cuenta los patrones culturales de la lengua de destino. Distinguiremos cinco aspectos dentro de la evaluación del entorno que rodea a la comunicación:

1) Rasgos personales de los participantes: por ejemplo, una relación cordial entre el presidente de un país poderoso y el presidente de un país pequeño y con pocos recursos viene condicionada por el hecho de que el primero controla la relación profesional de ambos. En caso de conflicto, el desequilibrio de poder que existe entre ambos se verá acentuado por los diferentes papeles que desempeñan.

2) Naturaleza y causa del conflicto: las diferentes creencias o sistemas de valores pueden dar lugar a conflictos cuando las personas que albergan esas diferencias compiten por objetivos diferentes de los cuales perciben que sólo podrá alcanzarse uno. Cuanto más comprometida esté la identidad personal y ciertos valores básicos, más probable será que se produzca un conflicto y más difícil resultará resolver las diferencias existentes. Por eso no es fácil traducir o interpretar entre distintas culturas acerca de temas religiosos, dilemas éticos y valores básicos (aborto, pena de muerte, oposición entre capitalismo y comunismo). 
Peñalver, E. A. - Retos éticos de los agentes comunicativos en situaciones de conflicto

3) Esclarecimiento de los objetivos de cada una de las partes: es necesario definir la naturaleza del conflicto para poder identificar los objetivos de las partes y actuar de acuerdo a ellos. Puede que bajo una forma de expresarse se esconda la intención de conseguir indirectamente un objetivo determinado. 4) Examen del clima en que se produce la comunicación: es necesario examinar en esta cuarta fase del proceso, el entorno en el que se produce la comunicación. De esta forma, el tipo de institución en la que nos encontremos, el factor tensión y los precedentes existentes respecto a situaciones conflictivas, son variables muy importes para definir cómo se debe abordar la transmisión del mensaje.

5) Decisión sobre la forma más adecuada de transmitir el mensaje de acuerdo a dicha situación de conflicto. Para ello hay que tener en cuenta una serie de aspectos, como establecer la comunicación con credibilidad, de forma que las partes puedan sentir también que el oponente está diciendo la verdad. El traductor establecerá también un nivel de confianza entre las dos partes, de modo que la transacción entre estas pueda avanzar hacia la regulación de las diferencias. Zand (1972: 234) define la confianza como la «regulación consciente de la dependencia mutua, que variará según la tarea a realizar, la situación y la otra persona». Para ello es necesario utilizar comunicaciones precisas y adecuadas, tratar de comprender por anticipado los intereses de las partes para poder transmitir el mensaje con la profundidad que las partes desean y así cumplir con su cometido y por último, buscar confirmación en todo momento de que las partes están comprendiendo el mensaje interpretado teniendo en cuenta la reacción y respuesta de esta. En este sentido, coincidimos con Carbonell (2004) en que conviene en primer lugar deshacerse de la noción de equivalencia, de considerar que lo que prima es la información del original y reproducirla salvando las dificultades gramaticales, semánticas o discursivas que plantea el texto original. De esta manera, la selección semántica será de gran importancia en una situación conflictiva. Así, el traductor deberá escoger cuidadosamente las palabras para que ambas 
Peñalver, E. A. - Retos éticos de los agentes comunicativos en situaciones de conflicto

partes entiendan el mensaje con claridad, optando por las que tengan un significado claramente denotativo y no uno connotativo que pueda entrañar la interpretación personal y subjetiva de los hechos por parte del interlocutor en función de las experiencias vividas. Esta posibilidad de introducir palabras que produzcan reacciones opuestas entre las personas involucradas puede pues establecer una barrera real para gestionar el conflicto. Es por ello que es importante tratar de prever qué palabras pueden empeorar el problema y evitar su uso en cuanto nos sea posible. Para reducir los riesgos de traducción imprecisa, el comunicador debe intentar conseguir un traductor cualificado y es por ello que la elección del mismo requiere especial cuidado. En este sentido, queremos señalar que un traductor no es cualquier persona que ha vivido en un país durante un tiempo determinado, sino aquellos que cuentan con las competencias traductológicas necesarias para evitar errores de traducción que afecten a una situación comunicativa cuyo desarrollo en una situación de conflicto pueda ser fundamental y que conozca los aspectos éticos de la profesión que se han mencionado para adoptar así la solución más adecuada.

\section{Caso práctico: análisis de resultados}

Nos detendremos a continuación a analizar los aspectos planteados en los apartados anteriores tomando como referencia el estudio llevado a cabo por Palmer (2007: 13-28), que hemos considerado de gran interés como caso práctico ilustrativo por la aplicabilidad que tienen en él nuestros enunciados. El estudio se centra en el rol del agente comunicativo como intermediario de la transmisión de información entre dos lenguas atendiendo a la situación conflictiva que se desarrollaba en Irak desde la invasión anglo-americana de 2003, desencadenada por los atentados contra las Torres Gemelas de Nueva York el 11 de septiembre de 2001. Tras este atentado, se puso de manifiesto 
Peñalver, E. A. - Retos éticos de los agentes comunicativos en situaciones de conflicto

la importancia de la traducción para evitar consecuencias calamitosas para la seguridad nacional. Apter $(2006,2009)$ reveló que el FBI había cometido en Estados Unidos importantes fallos de contrainteligencia por no haber contado con suficientes traductores de área. Del mismo modo, en octubre de 2001 el diario El País recogía las declaraciones de Michael Hayden, responsable de la Agencia de Seguridad Nacional (ASA), en las que afirmaba que su entidad había captado mensajes que podrían haber servido para alertar del inminente atentado si hubieran sido traducidos (Pegenaute 2010).

Sin embargo, y aunque se reconociera la importancia de la traducción para facilitar el tránsito de la información, las condiciones que se establecían alrededor de la profesión no eran de lo más favorecedoras:

- En cuanto al tema de la seguridad en Irak, hay que decir que desde marzo de 2003 a octubre de 2004, murieron asesinados 36 periodistas y trece acompañantes entre los que se incluían a los traductores (PALMER 2007). Además, aunque no los veamos en la televisión, con los soldados van los intérpretes y según la Federación Internacional de Traductores (FIT) después de los primeros, los intérpretes constituyen el mayor grupo de víctimas civiles de este conflicto (KAHANE 2007).

- El grado de confianza de los traductores se veía mermado cuando se alegaba que, entre los traductores locales contratados en Irak para trabajar en las fuerzas armadas de Estados Unidos, algunos habían aportado información falsa a los soldados. De este modo, se deducía que al no contar con un mensaje fielmente interpretado, la información periodística que se transmitía se veía influenciada por el papel del traductor.

Teniendo en cuenta estas observaciones preliminares, Palmer realizó una serie de entrevistas a periodistas de Francia y Reino Unido para determinar el grado de dependencia que tenían respecto al agente comunicativo. En sus comentarios, todos ellos afirmaron que confiaban plenamente en sus traductores, pero que eran conscientes de que un grado de 
Peñalver, E. A. - Retos éticos de los agentes comunicativos en situaciones de conflicto

dependencia elevado les podía hacer sufrir las consecuencias de una traducción mal hecha o de la omisión de datos importantes.

Ante esta situación descrita por los periodistas, surge la cuestión de dónde se sitúa la ética que el agente comunicativo tiene que contemplar en este tipo de situaciones para posicionarse como un profesional neutral ante ambas partes. De este modo, comprobamos a través de este estudio que los principios éticos de la traducción no se tenían en cuenta, ya que la mayoría de las personas que se contrataban en Irak para actuar como traductores no contaban con las competencias estándares que se requieren de los profesionales que han recibido formación. Esto viene de nuevo a corroborar nuestra afirmación de que no basta con ser bilingüe para poder desarrollar estratégicamente una situación comunicativa de conflicto. De esta forma, la práctica del ejercicio profesional de la traducción en Irak nos ofrece desafortunadamente más de un ejemplo de cómo todos los requisitos éticos enumerados con anterioridad y las propuestas metodológicas para abordar una situación comunicativa de conflicto de forma satisfactoria en dos lenguas se ignoran cuando no se cuenta con la formación necesaria. En este sentido, un traductor formado procedería antes de llevar a cabo su actividad a una preparación de la misma en función de los parámetros anunciados en el apartado de metodología. Así, tendría en cuenta la naturaleza y causa del conflicto, el contexto en el que se produce la comunicación según el lugar y la situación de los hablantes, para poder tomar las decisiones traductológicas más adecuadas a la hora de trasmitir el mensaje fielmente y asumiendo una posición neutral, con el único interés de comunicar una información y cumplir con su trabajo. De este modo, y según la naturaleza del mensaje, el agente comunicativo adoptaría el papel de transcodificador si bastara con trasmitir el mensaje, o de clarificador, si tuviera que dar un paso más interviniendo con alguna aclaración que facilitara la comprensión del mensaje debido a la existencia de conceptos que no son obvios para los europeos. Finalmente, el agente comunicativo podría intervenir en tanto que experto cultural cuando 
Peñalver, E. A. - Retos éticos de los agentes comunicativos en situaciones de conflicto

la aclaración debido a las diferencias entre culturas fuera necesaria para la comprensión total del mensaje por ambas partes. En el caso de la situación de conflicto en Irak, son estas dos últimas funciones las que se adoptan con mayor frecuencia. De este modo, si tenemos en cuenta que es necesario siempre que quede claro el mensaje para no agravar el desarrollo de un conflicto, será necesario la aclaración de muchos conceptos y la transgresión de barreras culturales que se imponen entre la cultura iraquí y la europea. Sin embargo, como se puede extraer de las entrevistas, el problema de contar con personas locales bilingües no formadas para llevar a cabo de forma profesional la actividad de traductor subyace porque no prevalece un concepto elevado de lo que significa traducir. Por lo tanto, a la hora de contar con los servicios de un traductor en una situación de conflicto, los periodistas no priman la formación específica que estos hayan podido recibir al respecto, sino que prefieren que estos sean más bien lo que se conoce en inglés como «fixer», es decir, que cuenten con una serie de contactos en el área local que les permitan obtener noticias que puedan tener un alto índice de impacto en los países en los que se publiquen.

\section{Conclusiones}

A modo de conclusión, cabe afirmar que coincidimos con Pegenaute (2010) en que la ingente cobertura periodística de los grandes atentados como el 11-S y conflictos como la guerra de Irak han servido, de forma indirecta, para llamar la atención sobre el papel destacado que cumplen los traductores en tanto que agentes comunicativos en situaciones de conflicto. De este modo, en esta comunicación hemos puesto de manifiesto la dificultad de su labor puesto que no solo se trata de transmitir un mensaje, sino de analizar también las principales connotaciones del discurso y transmitirlo eligiendo aquellas palabras que cuenten con un significado claramente denotativo. En 
PeÑAlVER, E. A. - Retos éticos de los agentes comunicativos en situaciones de conflicto

esta misma línea, hemos argumentado acerca de las dudas que surgen sobre su cometido cuando se trata de mantener una posición neutral, adoptando los principios éticos de su profesión. Así, hemos señalado que un traductor profesional formado al respecto sabrá analizar previamente la situación en función de los principios metodológicos propuestos para poder adoptar la función comunicativa que le corresponda según los requisitos de la situación y adoptar consecuentemente las estrategias traductológicas más adecuadas para transmitir la información fielmente. De esta forma, hemos enfatizado la necesidad de la formación para contar con agentes comunicativos que sepan desenvolverse de forma eficaz y correcta en este tipo de situaciones. Consideramos por otro lado que el desarrollo de este tipo de estudios sobre la sociología de la traducción, entendiéndola como actividad comunicativa entre dos lenguas y culturas en un determinado contexto social, y sobre sus principios éticos, resulta imprescindible conforme la globalización y los conflictos bélicos superan las fronteras lingüísticas, culturales y nacionales.

Sería deseable contar con un mayor acervo biográfico y testimonial de los traductores e intérpretes que nos permitiera estudiar sus hábitos de comportamiento y sus diferentes respuestas ante los retos y exigencias planteados por los distintos encargos de traducción. De esta forma, contribuiremos al desarrollo de estudios aplicados que formen un triángulo investigación-formación-profesión cuyos elementos se alimenten mutuamente para facilitar así la búsqueda de soluciones prácticas a los problemas reales que existen en la actualidad en la profesión y que satisfagan los requisitos de transmisión de información que se requieren de estos agentes comunicativos en determinadas situaciones de conflicto. 
Peñalver, E. A. - Retos éticos de los agentes comunicativos en situaciones de conflicto

\section{Referencias bibliográficas}

ABRIL, M. I. La Interpretación en los Servicios Públicos: Caracterización como género, contextualización y modelos de formación. Hacia unas bases para el diseño curricular. Tesis doctoral. Granada: Universidad de Granada, 2006.

ANDERSON, R. B. Perspectives on the role of interpreter. In: PöCHKACKER, F. y SHLESINGER, M. (Eds.), The Interpreting Studies Reader. Londres/Nueva York: Routledge, 1976/2002: 209-217.

APTER, E. Translation After 9/11: Mistranslating the Art of War. In: The Translation Zone: A New Comparative Literature. Princeton/Oxford: Princeton University Press, 2006: 12-22.

APTER, E. 9/11: Terrorism, Immigration, Language Politics. In: Bielsa, E. y Hugues, C. W. (Eds.), Globalization, Political Violence and Translation. Basingstoke/Nueva York: Palgrave McMillan, 2009: 195-206.

ARrojo, R. Oficina de tradução. A teoría na prática. São Paulo: Editora Ática, 1992.

BAKER, M. Translation and Conflict. A Narrative Account. Nueva York: Routledge, 2006.

Borisoff, D. y VICTOR, D. A. Gestión de conflictos. Un enfoque de las técnicas de comunicación. Madrid: Ediciones Díaz de Santos, 1991.

CAMBRIDGE, J. Interlocutor roles and the pressures on interpreters. In: VALERO, C. y MANCHO, G. (Eds.), Traducción e Interpretación en los Servicios Públicos: Nuevas Necesidades para Nuevas Realidades. Alcalá de Henares: Madrid, 2002.

CARBonelL, O. La ética del traductor y la ética de la traductología. In: Grupo TLS (Eds.), Ética y política de la traducción literaria. Málaga: Miguel Gómez Ediciones, 2004: 17-45.

Chesterman, A. Ethics of translation. In: Snell-HornBy, M. et al. (Eds.), Translation as Intercultural Communication. Amsterdam/Philadelphia: John Benjamins, 1995.

COPELAND, L. y GRIGSS, J. Going international: How to make friends and deal effectively in the global marketplace. Nueva York: Random House, 1985.

Coser, L. A. The Functions of Social Conflict. Nueva York: The Free Press, 1956. 
Peñalver, E. A. - Retos éticos de los agentes comunicativos en situaciones de conflicto

Douglas, M. Implicit Meanings. Londres: Routledge \& Kegan Paul Ltd, 1975.

HATIM, B. y MASON, I. The translator as a communicator. Nueva York: Routledge, 1997.

HURTADO, A. Enseñar a traducir. Metodología en la formación de traductores e intérpretes. Teoría y fichas prácticas. Madrid: Edelsa, 1999.

KAHANE, E. Intérpretes en conflictos: los límites de la neutralidad Disponible en: <http://goo.gl/SDj56H> (03/11/2014).

MAIER, C. The translator as an intervenient being. In: Munday, J. (Ed.), Translation as intervention. Londres: Continuum International Publishing Group, 2007: 1-17.

Mandelbaum, D. Edwar Sapir: Culture, Language and Personality. Bekerley: University of California Press, 1962.

PaLMER, J. Interpreting and Translation for Western Media in Iraq. In: SalamaCARR, M. (Ed.), Translating and interpreting conflict. Amsterdam/Nueva York: Rodopi, 2007: 13-28.

PegenAute, L. La traducción como herramienta preventiva y paliativa del terrorismo internacional. Disponible en: <http://goo.gl/bRtgzB > (03/11/2014).

SmIRnov, S. An overview of liaison interpreting. In: Perspectives: Studies in Translatology, 5(2), 1997: 211-226.

VIDAL, M. C. A. La Facultad de Traducción y Documentación analiza los discursos políticos en la era de la globalización. Disponible en: <http://goo.gl/i1FLC6>. (03/11/2014).

WHORF, B. L. Collected Papers on Metalinguistics. Washington: Department of State, Foreign Service Institute, 1952.

ZIMMAN, L. Intervention as a pedagogical problem in community interpreting. In: DollerUP, C.; LINDEGAARD, A. (Eds.), Teaching Translation and Interpreting 2 - Insights, Aims, Visions. Amsterdam/Philadelfia: John Benjamins, 1994: 217224.

Recebido em: 1 maio 2015

Aprovado em: 24 nov. 2015 